\title{
Utilidad de la Escala Multidimensional de Craving de Alcohol (EMCA) en la práctica clínica
}

\author{
Josep Guardia Serecigni; Elena luquero Vived; Núria Siñol llosa; Teresa Burguete; Maria Cardús Moya \\ Unidad de Conductas Adictivas. Servicio de Psiquiatría. Hospital de la Santa Creu i Sant Pau. Barcelona.
}

Enviar correspondencia a:

Josep Guardia Serecigni. Unidad de Conductas Adictivas. Hospital de la Santa Creu i Sant Pau. C/ San Antoni Maria Claret n 167.08025 - BARCELONA Telfs: 932919180 o 9329191 31. FAX: 9329191 78. E-mail: jguardia@santpau.es

Recibido: 20 de diciembre de 2005 Aceptado: 22 de abril de 2006.

\section{RESUMEN}

Introducción: El craving es un síntoma central de las conductas adictivas cuya intensidad suele relacionarse con la evolución del paciente. La escala EMCA ha demostrado ser un instrumento válido y fiable para su evaluación. El objetivo es analizar la utilidad de la escala en la evaluación de la eficacia y en la mejora de la planificación del tratamiento del alcoholismo.

Pacientes y método: Con una muestra de 151 pacientes con trastorno por dependencia de alcohol, se estudió la relación de la escala EMCA con la intensidad de la dependencia, la comorbilidad psiquiátrica y adictiva y con otras escalas utilizadas en la evaluación del alcoholismo. Finalmente, se evaluó la sensibilidad de la escala para detectar cambios en la evolución de los pacientes en un estudio de seguimiento a seis meses.

Resultados: La EMCA discrimina entre el nivel grave de intensidad de la dependencia y los niveles moderado $(p<0,001)$ y leve $(p<0,001)$, así como entre pacientes con y sin comorbilidad adictiva $(p=0,011)$. El factor "deseo de beber" de la EMCA discrimina entre pacientes con y sin comorbilidad psiquiátrica $(p=0,041)$. La EMCA muestra una buena sensibilidad al cambio en el consumo de alcohol a lo largo del seguimiento.

Conclusiones: La intensidad del craving parece estar modulada por la intensidad de la dependencia del alcohol y la comorbilidad psiquiátrica y adictiva. La EMCA ha demostrado ser sensible a los cambios en la evolución del curso de los pacientes alcohólicos, y por tanto, ser útil en la planificación y la evaluación de los resultados del tratamiento del alcoholismo.

Palabras clave: Craving, Alcoholismo, Utilidad clínica, Diagnóstico del alcoholismo, Tratamiento del alcoholismo.

\section{ABSTRACT}

Introduction: Craving is a central symptom of addictive behaviors. Craving levels are usually related to patient's evolution. The MACS scale has prove a valid and reliable instrument to assess this symptom. The aim of this study is to test the usefulness of MACS to assess the efficacy of treatment and to improve the alcoholism treatment planning.

Patients and Method: A sample of 151 alcohol-dependence patients has been used to study the relationship between the MACS scale and dependence severity, psychiatric and addictive comorbidity and other alcoholism scales. Furthermore, the sensitivity of MACS to detect changes in patient's evolution during a six months follow-up study has been assessed.

Results: The MACS discriminate between the severe level of dependence severity and moderate $(p<0.001)$ and slight levels $(p<0.0001)$, as well as between patients with and without addictive comorbidity. The "desire to drink" factor of the MACS discriminate between patients with and without psychiatric comorbidity $(p=0.041)$. The MACS show a good sensitivity to detect changes in alcohol consumption during the follow-up study.

Conclusions: The craving severity seems to be modulated by the alcohol-dependence severity and psychiatric and addictive comorbidity. The MACS is sensitive to the changes that happen during the recovery process of alcoholism. Therefore it can be a useful instrument for alcoholism treatment planning and outcome treatment assessment.

Key words: Craving, Alcoholism, Clinical usefulness, Alcoholism diagnosis, Alcoholism treatment.

\section{INTRODUCCIÓN}

E craving ha demostrado ser uno de los síntomas centrales de toda conducta adictiva, 2, 3. Varios estudios han evaluado el rol del craving tanto en el resultado del tratamiento, como en la recaída ${ }^{4}$. Numerosas investigaciones han utilizado igualmente la evaluación del craving durante el tratamiento como indicador de la eficacia de las intervenciones farmacológicas ${ }^{5,6}$.

Existe una gran heterogeneidad en las definiciones del craving. Éste es conceptualizado de manera diferente en función del modelo teórico de aproximación 
a dicho fenómeno7,8. Aún así, existe cierto consenso acerca de definirlo como un estado emocional subjetivo de deseo intenso de beber o de auto-administrarse una determinada sustancia adictiva ${ }^{9}$ y como un fenómeno psicobiológico en el que inciden una gran diversidad de aspectos situacionales, motivacionales, cognitivos, emocionales, conductuales y de personalidad ${ }^{10}$.

El craving es crucial para comprender tanto el mantenimiento de la conducta adictiva en pacientes que no han dejado de beber, como la recaída en pacientes abstinentes ${ }^{11}$.

La intensidad del craving suele relacionarse con la evolución del paciente durante el proceso de recuperación y con otras variables psicopatológicas. La expresión del craving puede estar influenciada por la presencia de trastornos psiquiátricos como la depresión o la ansiedad ${ }^{12}$. Diversos estudios han comprobado que cuando la dependencia de sustancias va asociada a comorbilidad psiquiátrica ${ }^{13,14}$ o a determinados rasgos de personalidad como ansiedad o búsqueda de novedad ${ }^{15,3}$, el craving puede alcanzar una mayor intensidad. Otros estudios, sin embargo, no encuentran ésta relación ${ }^{2}$. Se ha estudiado incluso la eficacia de las medicaciones anti-craving en el tratamiento del alcoholismo asociado a comorbilidad psiquiátrica ${ }^{16}$.

Por otro lado, ha sido también examinada la relación entre la respuesta ante estímulos condicionados al alcohol y la intensidad de la dependencia ${ }^{17}$ y entre el craving y la intensidad de la dependencia ${ }^{2}$, encontrando una correlación significativa y positiva entre ambas variables.

Aunque se ha demostrado que el craving juega un rol principal en la recaída, existen muy pocos instrumentos de medida válidos y fiables desarrollados en nuestro país y la mayoría de ellos se basan en adaptaciones de escalas extranjeras ${ }^{10}$.

Dado que el concepto de craving hace referencia a un estado emocional subjetivo que da lugar a la búsqueda y consumo de la sustancia psicoactiva, tal y como ha ocurrido en otros estados subjetivos, éste debería ser descrito por aquellos que lo han experimentado ${ }^{18}$. En este sentido, la Escala Multidimensional de Craving de Alcohol (EMCA), construida esencialmente a partir de las verbalizaciones de los pacientes, ha demostrado ser un instrumento válido y fiable para la evaluación del craving y puede ser de gran utilidad en el diagnóstico y tratamiento de los pacientes alcohólicos ${ }^{10}$.

La información derivada de escalas como la EMCA puede suponer una mejora tanto para el proceso de recuperación del paciente (mejorando el insight del propio craving) como para la toma de decisiones terapéuticas en el desarrollo y la adaptación continua de las estrategias de prevención de recaídas a la evolución del paciente, por parte del clínico. Cuando la intensidad del craving del paciente no experimenta una disminución progresiva durante el tratamiento o ésta intensidad se reagudiza cuando éste ya había empezado a mejorar, el profesional puede estar ante un predictor de recaída o un indicador de mayor necesidad de tratamiento.

El objetivo del presente estudio es analizar la utilidad de la escala EMCA en la mejora de la evaluación de la eficacia y de la planificación del tratamiento del alcoholismo en la práctica clínica habitual. Para ello se ha estudiado (1) la relación de la escala con los diversos grados de intensidad de la dependencia de alcohol, la presencia de comorbilidad psiquiátrica y adictiva y con otras escalas utilizadas en la evaluación del alcoholismo. Así mismo, se ha realizado (2) una primera aproximación a los criterios de intensidad del craving en base a los percentiles obtenidos de las puntuaciones de la EMCA en función de la situación de consumo de los pacientes, para la interpretación clínica de las puntuaciones en dicha escala. Finalmente, se ha evaluado (3) la sensibilidad de la escala para detectar cambios en la evolución de los pacientes en un estudio de seguimiento a seis meses.

\section{MATERIAL Y MÉTODOS}

\section{Participantes}

La muestra se compuso de 151 pacientes que presentaban un trastorno por dependencia de alcohol como diagnóstico principal y que realizaban tratamiento en régimen de ingreso hospitalario para desintoxicación ( $n=34$ ) o se hallaban en tratamiento ambulatorio ( $n=117$ ) de su dependencia, en la Unidad de Conductas Adictivas del Hospital de la Santa Creu i Sant Pau de Barcelona.

\section{Procedimiento}

El estudio se llevó a cabo entre mayo del 2004 y enero del 2005. Los pacientes fueron invitados a participar en el estudio siempre y cuando no presentaran síntomas de intoxicación durante la evaluación ni un deterioro cognitivo diagnosticado. Todos los participantes firmaron el consentimiento informado antes de su inclusión en el estudio. En una primera fase (Basal) ( $n=$ 148) se realizó una recogida de datos socio-demográficos, de situación actual e historia de consumo de alcohol y de intervenciones farmacológicas y psicológicas actuales. Posteriormente se administró a los pacientes una batería de escalas para la evaluación de diferentes variables de la dependencia de alcohol. En una segunda fase ( $\mathrm{S} 1$ ) ( $\mathrm{n}=70)$, al mes de la visita Basal, se valoró la frecuencia (número de días de consumo) e inten- 
sidad (Unidad de Bebida Estándar -U.B.E- por día de consumo) del consumo de alcohol de los pacientes y se les administró una selección de dos escalas de las previamente administradas en la visita Basal. A los tres meses de la visita Basal se realizó un segundo seguimiento (S3) ( $n=48$ ), donde se volvió a valorar la frecuencia e intensidad del consumo de alcohol y se administraron las mismas escalas que en S1. A los seis meses de la visita Basal se realizó un tercer seguimiento (S6) ( $n=27$ ) dónde se llevó a cabo el mismo procedimiento que en S1 y S3.

Para ulteriores análisis, se clasificó a los pacientes en dos grupos. En el grupo de pacientes abstinentes se incluyeron aquellos pacientes que no realizaron ningún consumo de alcohol durante el tiempo de seguimiento que permanecieron en el estudio. En el caso de los pacientes que sólo acudieron a la visita Basal, se incluyeron aquellos que llevaban más de 20 días sin beber. En el grupo de pacientes con algún consumo fueron incluidos aquellos pacientes que realizaron algún consumo de alcohol en algún momento del seguimiento. De los pacientes que sólo acudieron a la visita Basal, se incluyeron los que llevaban menos de 20 días sin beber

\section{Instrumentos}

En la visita Basal se administraron las escalas en el siguiente orden: Escala de la Intensidad de la Dependencia al Alcohol (EIDA) ${ }^{19}$, Escala Visual Analógica de Craving de alcohol (EVA), Escala Multidimensional de Craving de Alcohol (EMCA) ${ }^{10}$ y Escala de los Componentes Obsesivo-Compulsivos de la Bebida $(\mathrm{CCOCB})^{20}$. En las fases de seguimiento S1, S3 y S6 se administraron las escalas EVA y EMCA en este mismo orden.

\section{Análisis de datos}

La relación de la escala EMCA con la intensidad de la dependencia de alcohol, se examinó mediante pruebas de análisis de la variancia de un factor (ANOVA), usando análisis post-hoc aplicando la corrección de Bonferroni para llevar a cabo las comparaciones entre los tres niveles de intensidad de dependencia establecidos por la escala EIDA ( $<21$ : dependencia leve; $21-$ 37: dependencia moderada; $>37$ dependencia grave).

La relación de la escala EMCA con la presencia de comorbilidad psiquiátrica y adictiva, fue calculada utilizando pruebas t-test y la prueba no paramétrica $U$ de Mann-Whitney en aquellas ocasiones donde la muestra era inferior a 30.

La relación de la escala EMCA con la EVA y el CCOCB fue valorada mediante el cálculo de coeficientes de correlación de Pearson.
Así mismo, se tabularon para distintas situaciones de consumo (muestra global, abstinencia y algún consumo) y para cada uno de los factores de la EMCA, las puntuaciones correspondientes a los percentiles 5 , $10,25,50,75,90$ y 95.

La sensibilidad de la escala para detectar cambios en la evolución de los pacientes fue valorada mediante el cálculo de coeficientes de correlación de Pearson con la frecuencia e intensidad del consumo de alcohol en S1, S3 y S6. Además se realizaron pruebas t-test (o prueba $U$ de Mann-Whitney) utilizando la situación del paciente con relación a su consumo de alcohol (abstinencia versus algún consumo de alcohol) en las cuatro visitas llevadas a cabo en el estudio, como variable independiente, y la puntuación total de la escala EMCA administrada en éstas cuatro visitas, como variable dependiente.

El procesamiento y el análisis de datos se realizó mediante el paquete estadístico SPSS 11.5 para Windows.

\section{RESULTADOS}

\section{Muestra}

La muestra total del estudio fue de 151 pacientes $(67,5 \%$ varones y $32,5 \%$ mujeres) con una edad media \pm DT (Mín-Máx.) de 47,3 \pm 9,8 (24-76) años. La mayor parte de la muestra estaba casada o vivía en pareja $(49,7 \%)$, tenía estudios primarios completos $(40,7 \%)$ y se hallaba en un periodo de consumo activo de alcohol activo o llevaba menos de 20 días sin beber $(55,6 \%)$.

Respecto a la comorbilidad psiquiátrica, un 47\% de la muestra presentaba alguna patología psiquiátrica concomitante al alcoholismo: trastorno del estado de ánimo (16,1\%), trastorno de personalidad (12,8\%) trastorno de ansiedad (6\%), trastorno ansioso-depresivo $(5,4 \%)$, trastorno psicótico $(4,7 \%)$ y trastorno por déficit de atención con hiperactividad $(3,4 \%)$.

Respecto a la comorbilidad adictiva, un 76,5\% presentaba algún otro trastorno por uso de sustancias psicoactivas concomitante al alcoholismo: nicotina (72,5\%), sedantes, hipnóticos o ansiolíticos (12,8\%), estimulantes $(10,7 \%)$, cánnabis $(10,7 \%)$, opiáceos $(4 \%)$ y otras conductas adictivas (4\%).

En relación al consumo de alcohol, la media de años de evolución del consumo fue de 26,4 $\pm 10,6$ (2-57) años. El consumo habitual diario de alcohol fue de 20,2 \pm 17,1 (2-160) U.B.E./día. La intensidad de la dependencia de alcohol (en función de la escala EIDA) fue leve en 43 casos (28,9\%), moderada en 54 casos $(36,2 \%)$ y grave en 52 casos $(34,9 \%)$. 
En relación a las intervenciones farmacológicas en el momento de la visita Basal, el 42\% ( $n=63$ ) tomaba algún fármaco para el tratamiento del alcoholismo (22\% interdictores, $11,3 \%$ anticraving y $9,3 \%$ distraneurine) y el $66,7 \%$ se hallaba igualmente en tratamiento con algún psicofármaco $(42,7 \%$ antidepresivos, 33,3\% benzodiacepinas, 19,3\% eutimizantes y $17,3 \%$ antipsicóticos).

El 41,3\% ( $n=62$ ) estaba recibiendo algún tipo de intervención psicológica relacionada con la adicción (91,8\% grupo terapéutico, 6,6\% hospital de día y $1,6 \%$ psicoterapia en otros centros) y el $45,3 \%$ realizaba determinaciones periódicas de sustancias en la orina (urinoanálisis).

\section{Relación entre el craving (EMCA) y la intensidad de la dependencia de alcohol (EIDA)}

Existen diferencias significativas en la puntuación total de la escala EMCA en función de los niveles de la intensidad de la dependencia de alcohol establecidos por la escala EIDA $(F(2,143)=17,42 ; p<0,001)$. Los pacientes con una dependencia grave refieren una mayor intensidad del craving $(35,7 \pm 14,6)$ que los pacientes con una dependencia moderada $(26,1 \pm 12,4)$ $(p<0,001)$ o leve $(20,6 \pm 9,8)(p<0,001)$. (Figura 1).

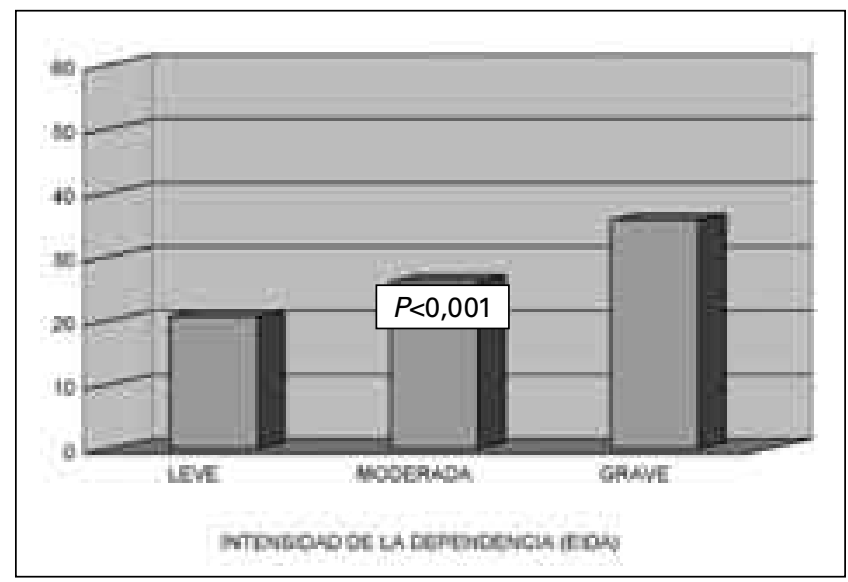

Figura 1: Media de la puntuación total de la escala EMCA en función de los niveles de intensidad de la dependencia delimitados por la escala EIDA

Concretamente existen diferencias significativas en el factor "deseo de beber" de la EMCA $(F(2,143)=19,56$ $\mathrm{p}<0,001)$, siendo los pacientes con una dependencia grave los que refieren un mayor "deseo" $(30,2 \pm 12,8)$ que los pacientes con una dependencia moderada $(21,2$ $\pm 10,6)$ o leve $(16,5 \pm 8,0)$.

En relación al factor "desinhibición conductual" de la EMCA, existe una tendencia a la significación estadística entre el nivel de dependencia grave y leve $(p=0.071)$. Los pacientes con una dependencia grave tienden a puntuar más elevado $(5,40 \pm 3,1)$ que los pacientes con una dependencia leve $(4,1 \pm 2,8)$.

\section{Relación entre el craving (EMCA) y la comorbili- dad psiquiátrica.}

No existen diferencias estadísticamente significativas aunque sí una tendencia a la significación (ttest $(143)=-1,89 ; p=0,061)$ en la puntuación total de la escala EMCA administrada en la visita Basal entre los pacientes con y sin comorbilidad psiquiátrica. Los pacientes que presentan comorbilidad psiquiátrica tienden a referir menor craving de alcohol $(25,3 \pm 13,3)$ que los pacientes que no la presentan $(29,6 \pm 14,3)$. En relación al factor "deseo de beber", los pacientes que presentan comorbilidad psiquiátrica refieren un menor "deseo" $(20,6 \pm 11,4)$ que los pacientes que no presentan dicha comorbilidad $(24,7 \pm 12,5)$ (t-test $(143)=-2,06 ; p=0,041)$. Éstas diferencias no se mantienen para el factor "desinhibición conductual" de la EMCA administrada en la visita Basal, ni para la EMCA administrada en las sucesivas visitas de seguimiento.

\section{Relación entre el craving (EMCA) y la comorbili- dad adictiva.}

Los pacientes que presentan comorbilidad adictiva concomitante a la dependencia de alcohol refieren una mayor intensidad del craving de alcohol en la escala EMCA administrada en la visita Basal $(29,0 \pm 14,5)$ que los pacientes que no presentan dicha comorbilidad $(23,0 \pm 10,8)$ (t-test $(72,70)=2,60 ; p=0,011)$. Respecto el factor "deseo de beber", los pacientes que presentan comorbilidad adictiva refieren un mayor "deseo" $(24,1 \pm 12,6)$ que los pacientes que no la presentan $(18,6 \pm 9,3)$ (t-test $(73,75)=2,75 ; p=0,008)$. Éstas diferencias no se mantienen para el factor "desinhibición conductual" de la EMCA administrada en la visita Basal, ni para la EMCA administrada en las sucesivas visitas de seguimiento.

Cuando se analizan éstas diferencias excluyendo la adicción a la nicotina, la puntuación total de la escala EMCA administrada en la visita Basal muestra también diferencias significativas (t-test $(60,69)=2,24$; $p=0,029)$. Los pacientes con algún trastorno por abuso o dependencia de sustancias psicoactivas diferentes a la nicotina refieren un craving de alcohol más elevado en la $\operatorname{EMCA}(32,1 \pm 16,1)$ que los que no presentan dicha comorbilidad adictiva $(25,8 \pm 12,7)$. Estas diferencias tampoco se mantienen en las sucesivas visitas de seguimiento. 
Relación de la escala EMCA con la EVA y el CCOCB.

La Tabla 1 muestra las correlaciones de Pearson entre la puntuación total y de los dos factores de la EMCA, con la EVA, con las dos sub-escalas del CCOCB y con la puntuación total del CCOCB. La puntuación total de la escala EMCA y su factor "deseo de beber" muestran unas correlaciones elevadas y significativas con la EVA, con la puntuación total del CCOCB y con sus dos sub-escalas de componentes obsesivo y compulsivo. El factor "desinhibición conductual" de la EMCA muestra unas correlaciones significativas pero moderadas tanto con la EVA como con las puntuaciones del CCOCB.

Tabla 1. Correlaciones de Pearson entre la escala EMCA y las escalas EVA y CCOCB

\begin{tabular}{|l|c|c|c|}
\hline & \multicolumn{2}{|c|}{ EMCA } & Total \\
\hline EVA & Deseo de beber & Desinhibición Conductual & $0,76^{* *}$ \\
\hline CCOCB - Total & $0,77^{* *}$ & $0,50^{* *}$ & $0,85^{* *}$ \\
\hline CCOCB - Obsesivo & $0,83^{* *}$ & $0,62^{* *}$ & $0,77^{* *}$ \\
\hline CCOCB - Compulsivo & $0,77^{* *}$ & $0,50^{* *}$ & $0,82^{* *}$ \\
\hline
\end{tabular}

EMCA, Escala Multidimensional de Craving de Alcohol

EVA, Escala Visual Analógica de Craving de Alcohol

CCOCB, Cuestionario de los Componentes Obsesivo-Compulsivo de la Bebida

* $P \leq 0,01$

\section{Grados de intensidad del craving establecidos por la EMCA}

En la Tabla 2 se presentan diferentes criterios de intensidad del craving en base a los percentiles obtenidos para cada factor de la EMCA y para la puntuación total en función de la situación de consumo (muestra global, abstinencia y algún consumo). Estos criterios pueden interpretarse tentativamente como una aproximación a los valores de referencia a utilizar para valorar las puntuaciones de la EMCA en la práctica clínica.

Tabla 2. Interpretación de las puntuaciones de la EMCA en función de la situación de consumo (abstinencia vs algún consumo)

\begin{tabular}{|c|c|c|c|c|}
\hline & & \multicolumn{3}{|c|}{ CRAVING (EMCA) } \\
\hline & & Leve $^{a}$ & Moderado & Intenso ${ }^{\circ}$ \\
\hline \multirow[t]{3}{*}{ ABSTINENTES } & Deseo de beber & $<11$ & $11-15$ & $>15$ \\
\hline & $\begin{array}{l}\text { Desinhibición } \\
\text { conductual }\end{array}$ & $<3$ & 3 & $>3$ \\
\hline & Total & $<13$ & $13-20$ & $>20$ \\
\hline \multirow[t]{3}{*}{ ALGÚN CONSUMO } & Deseo de beber & $<21$ & $21-39$ & $>39$ \\
\hline & $\begin{array}{l}\text { Desinhibición } \\
\text { conductual }\end{array}$ & $<5$ & $5-8$ & $>8$ \\
\hline & Total & $<27$ & $27-46$ & $>46$ \\
\hline \multirow[t]{3}{*}{ MUESTRA GLOBAL } & Deseo de beber & $<12$ & $12-33$ & $>33$ \\
\hline & $\begin{array}{l}\text { Desinhibición } \\
\text { conductual } \\
\end{array}$ & $<3$ & $3-7$ & $>7$ \\
\hline & Total & $<16$ & $16-40$ & $>40$ \\
\hline
\end{tabular}

a puntuaciones por debajo del percentil 25 en la escala EMCA

${ }^{b}$ puntuaciones entre los percentiles 25 y 75 en la escala EMCA

${ }^{c}$ puntuaciones por encima del percentil 75 en la escala EMCA 
La figura 2 muestra la evolución de las puntuaciones medias en la EMCA a lo largo del seguimiento en función de los criterios de intensidad del craving.

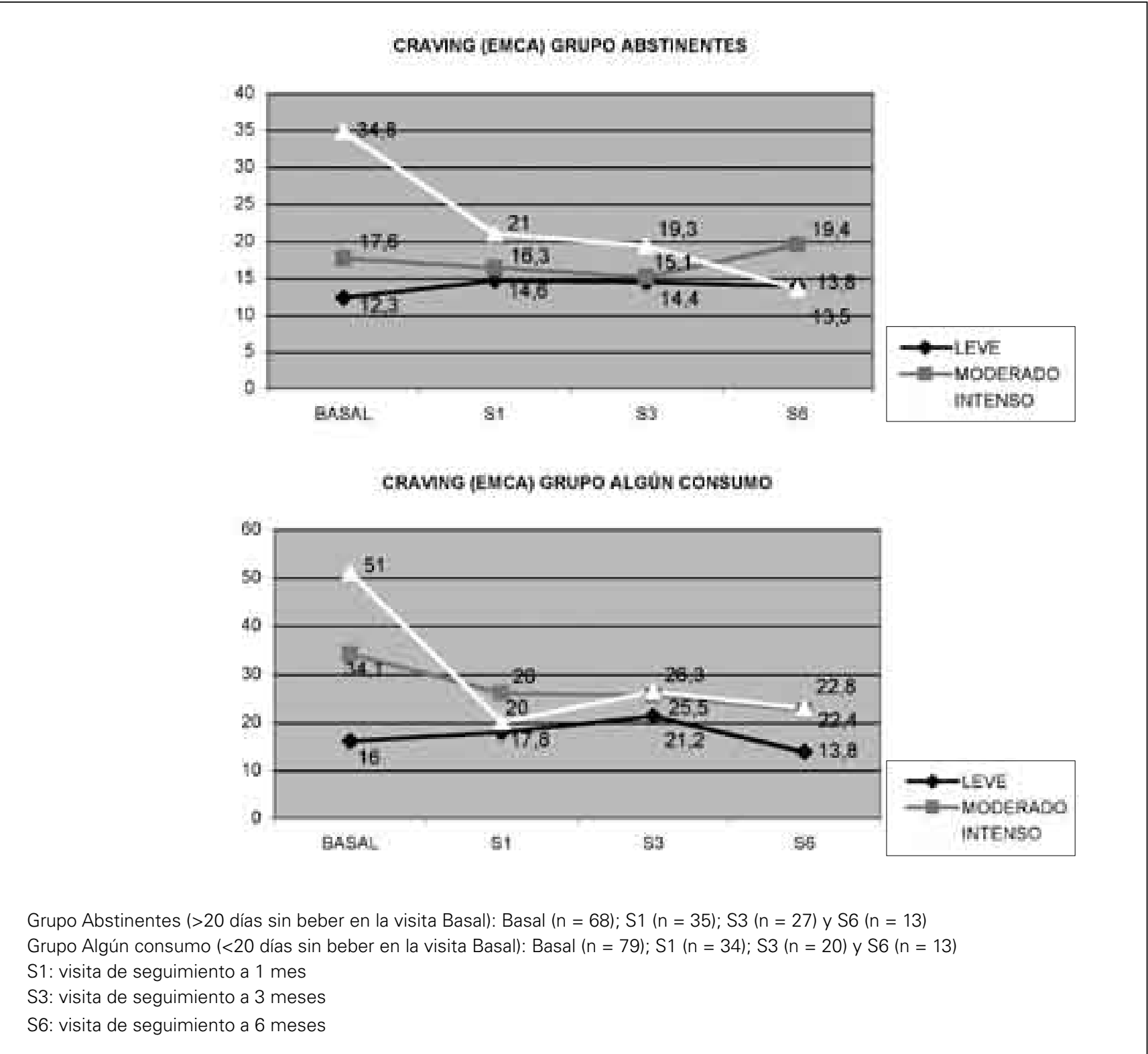
Figura 2. Evolución de las puntuaciones medias en la escala EMCA en función de los criterios
de intensidad del craving según sean abstinentes o con algún consumo

\section{Sensibilidad de la EMCA}

EMCA y situación del consumo de alcohol: abstinentes versus algún consumo de alcohol

La Tabla 3 muestra las puntuaciones medias y las desviaciones típicas obtenidas por los pacientes (abstinentes vs algún consumo de alcohol) en la escala EMCA administrada en la visita Basal y en las sucesivas visitas de seguimiento. Existen diferencias signifi- cativas en la puntuación total de la EMCA administrada en la visita Basal (t-test $(144,20)=6,53 ; p<0,001)$, en S1 (t-test $(48,93)=2,73 ; p=0,009)$, S3 (t-test $(22,70)=2,48$; $\mathrm{p}=0,021)$ y $\mathrm{S} 6(\mathrm{U}-\mathrm{MW}=50,0 ; \mathrm{p}=0,042)$ entre el grupo de pacientes abstinentes y el grupo de pacientes con algún consumo. La mayor intensidad del craving fue observada en aquellos pacientes que realizaron algún consumo de alcohol en algún momento del programa de seguimiento comparado con el grupo de pacientes que mantuvieron la abstinencia (Figura 3). 
Tabla 3. Puntuaciones en la escala EMCA en función de la situación de consumo de alcohol de los pacientes

\begin{tabular}{|c|c|c|}
\hline & $\begin{array}{l}\text { Algún consumo de } \\
\text { alcohol }\end{array}$ & Abstinentes \\
\hline EMCA administrada en la visita Basal $(n=148)$ & $33,5 \pm 13,6$ & $20,5 \pm 10,6$ \\
\hline EMCA administrada en S1 $(n=70)$ & $22,6 \pm 11,0$ & $16,9 \pm 5,7$ \\
\hline EMCA administrada en S3 ( $n=48)$ & $24,6 \pm 14,8$ & $16,0 \pm 5,4$ \\
\hline EMCA administrada en S6 $(n=27)$ & $19,8 \pm 7,9$ & $15,6 \pm 6,5$ \\
\hline
\end{tabular}

S1: visita de seguimiento a un mes. S3: visita de seguimiento a tres meses. S6: visita de seguimiento a 6 meses. $X \pm D T$

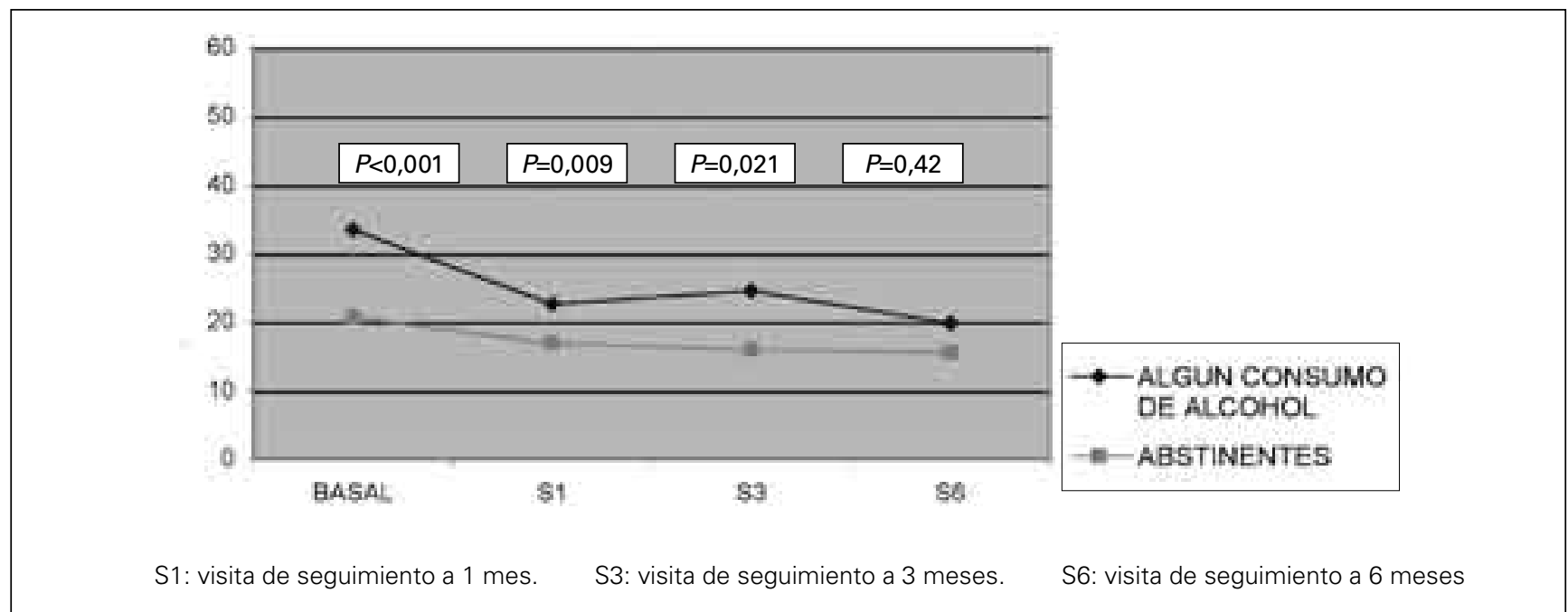

Figura 3: Evolución de las puntuaciones medias en la escala EMCA a lo largo del periodo de seguimiento

\section{EMCA y frecuencia e intensidad del consumo de} alcohol

La Tabla 4 muestra las correlaciones de Pearson entre las puntuaciones de la escala EMCA administrada en las tres visitas de seguimiento y los correspon- dientes registros de la frecuencia y la intensidad del consumo de alcohol. En relación a la frecuencia, todas las correlaciones son significativas excepto para el factor "desinhibición conductual" en S3. En relación a la intensidad del consumo, existen únicamente correlaciones significativas en S1.

Tabla 4. Correlaciones de Pearson entre la escala EMCA y la intensidad y la frecuencia del consumo de alcohol en S1, S3 y S6

\begin{tabular}{|c|c|c|c|}
\hline & \multicolumn{3}{|c|}{ EMCA 1 mes $(n=70)$} \\
\hline & Deseo & Desinhibición Conductual & Total \\
\hline \% días sin consumo de alcohol en S1 & $-0,31^{* *}$ & $-0,26^{*}$ & $-0,34 * *$ \\
\hline \multirow[t]{3}{*}{ consumo de alcohol en U.B.E./día de consumo en S1 } & $0,26^{*}$ & $0,32 * *$ & $-0,31 * *$ \\
\hline & \multicolumn{3}{|c|}{ EMCA 3 meses $(n=48)$} \\
\hline & Deseo & Desinhibición Conductual & Total \\
\hline \% días sin consumo de alcohol en S3 & $-0,37^{* *}$ & $-0,27$ & $-0,37 * *$ \\
\hline \multirow[t]{3}{*}{ consumo de alcohol en U.B.E./día de consumo en S3 } & 0,21 & 0,15 & 0,21 \\
\hline & \multicolumn{3}{|c|}{ EMCA 6 meses $(n=27)$} \\
\hline & Deseo & Desinhibición Conductual & Total \\
\hline \% días sin consumo de alcohol en S6 & $-0,45^{*}$ & $-0,71 * *$ & $-0,55 * *$ \\
\hline consumo de alcohol en U.B.E./día de consumo en S6 & 0,12 & 0,19 & 0,14 \\
\hline
\end{tabular}

\footnotetext{
S1: visita de seguimiento a un mes.

S3: visita de seguimiento a tres meses.

S6: visita de seguimiento a 6 meses

${ }^{*} \mathrm{P}<0,05$. ** $\mathrm{P} \leq 0,01$.
} 


\section{DISCUSIÓN}

El presente estudio ha demostrado la utilidad clínica de la escala EMCA en la práctica clínica diaria para la valoración de una variable de carácter subjetivo tan relevante como el craving en el tratamiento de la dependencia de alcohol.

La escala EMCA es capaz de discriminar a través de la puntuación total o del factor "deseo de beber" entre el nivel de dependencia grave y los niveles de dependencia moderada y leve. El profesional deberá tomar en consideración que el craving experimentado por los pacientes con una dependencia de alcohol grave puede interferir de manera más incisiva en el proceso de recuperación. Otros estudios han destacado que tener una medida del craving de los pacientes puede proporcionar un buen indicador de la gravedad de su dependencia de alcohol ${ }^{21}$.

En cuanto a la comorbilidad psiquiátrica asociada al alcoholismo, han sido los pacientes que presentan éste tipo de comorbilidad los que refieren menor "deseo de beber", observándose también una tendencia en la misma dirección en la puntuación total de la EMCA. Este resultado podría estar indicando una posible dificultad de insight o de reconocimiento del craving en este tipo de pacientes. La valoración del craving podría proporcionar información en relación a la capacidad que el paciente tiene para reconocer y monitorizar sus estados internos relacionados con la conducta de beber ${ }^{21}$ y por lo tanto, servir al clínico para detectar aquellos pacientes que presentarán una mayor dificultad en la adquisición y puesta en práctica de estrategias de afrontamiento adecuadas. Por otro lado, este resultado va en la dirección contraria de otros estudios donde se relaciona ésta comorbilidad con una mayor intensidad del craving ${ }^{13,14}$.

En la valoración de la comorbilidad adictiva concomitante al alcoholismo, la EMCA parece poder detectar diferencias en los niveles de craving entre pacientes con y sin este tipo de comorbilidad. Parece, por tanto, que la dependencia o abuso de otras sustancias psicoactivas cursa con una mayor sensación subjetiva del craving de alcohol. Cuando se excluye del análisis la dependencia de nicotina (la más ampliamente representada en la presente muestra), la escala sigue detectando dichas diferencias.

Los resultados de las pruebas de correlación entre la escala EMCA y las escalas EVA y CCOCB muestran lo ya corroborado en el estudio de validación de la EMCA ${ }^{10}$, siendo el factor "deseo de beber" y la puntuación total de la EMCA, en contraste con la "desinhibición conductual", las escalas que parecen relacionarse más directamente con el componente obsesivo-compulsivo de la conducta de beber.

Por otra parte, la escala EMCA (en base a las cuatro administraciones en la visita Basal, S1, S3 y S6) ha demostrado ser sensible a los cambios en la evolución del paciente alcohólico en un programa de seguimiento a seis meses, siendo capaz de diferenciar entre pacientes que están abstinentes o que se mantendrán abstinentes a lo largo del seguimiento y pacientes que están en consumo de alcohol activo o harán algún consumo de alcohol en algún momento del seguimiento. La escala puede ser, por tanto, utilizada por el clínico como un buen indicador de la situación actual o evolución del paciente. Dicho indicador es especialmente relevante a la hora de la toma de decisiones terapéuticas en el sentido de adaptar de manera dinámica e individualizada las estrategias de intervención así como la intensidad y duración del tratamiento. Al tratarse de una escala de estado, la EMCA debe demostrar ser sensible con mayor necesidad que otras escalas. Nuestro estudio corrobora los resultados obtenidos en el estudio de validación previo de la $\mathrm{EMCA}^{10}$, demostrando la capacidad de la escala para detectar cambios clínicos en la evolución de los pacientes. Ésta sensibilidad a los cambios, junto a su sencillez y rápida evaluación, la convierten en un instrumento idóneo para la valoración del estado del paciente así como de la eficacia de las intervenciones farmacológicas para el tratamiento del alcoholismo que éste está recibiendo.

Aunque la escala EMCA ha sido ya utilizada como escala de referencia en el estudio de otras escalas relacionadas con el autocontrol ${ }^{22}$, existen ciertas limitaciones en el presente estudio que hacen necesario seguir desarrollando investigaciones para confirmar determinadas propiedades de dicha escala. En primer lugar, nos hemos encontrado con un porcentaje de abandonos elevado, aunque esperable en los estudios longitudinales ${ }^{23,24}$. De la muestra de 151 pacientes con dependencia de alcohol de la que se parte en la visita Basal se deriva un reducido tamaño de la muestra en S6 (82\% de abandonos). Por tal motivo se han utilizado pruebas no paramétricas para el análisis estadístico de los datos, evidenciando la necesidad de corroboración de los resultados encontrados en ese momento temporal en nuevos estudios.

Aunque los resultados del estudio parecen apuntar que el craving de alcohol podría estar modulado por la comorbilidad tanto psiquiátrica como adictiva, igualmente sería necesario analizar ésta influencia con una muestra más amplia y heterogénea y, por tanto, más representativa del grupo de pacientes con comorbilidad asociada a la dependencia de alcohol.

En definitiva, la EMCA parece ser un instrumento breve, rápido y de fácil corrección, características que pueden convertirla en una herramienta de evaluación de uso generalizado y habitual en el diagnóstico y tratamiento de los pacientes que presentan un trastorno por dependencia de alcohol, tanto en la práctica clínica habitual como en los ensayos clínicos u otro tipo de investigación sobre el tratamiento del alcoholismo. 


\section{AGRADECIMIENTOS}

Los autores agradecemos la colaboración a todos los profesionales y pacientes de la Unidad de Conductas Adictivas del Hospital de la Santa Creu i Sant Pau de Barcelona que han colaborado con el estudio.

Agradecemos también el apoyo al Institut de Recerca del Hospital de la Santa Creu i Sant Pau de Barcelona, así como la financiación parcial del estudio a Jansen-Cylag.

\section{REFERENCIAS}

1. Goldstein RZ, Volkow ND. Drug addiction and its underlying neurobiological basis: neuroimaging evidence for the involvement of the frontal cortex. Am J Psychiatry 2002; 159: 1642-52.

2. Drummond DC, Phillips TS. Alcohol urges in alcoholdependent drinkers: further validation of the Alcohol Urge Questionnaire in an untreated community clinical population (Abstract). Addiction 2002; 97: 1465-72.

3. Tavares H, Zilberman ML, Holdings DC, el-Guelbay N. Comparison of craving between pathological gamblers and alcoholics. Alcohol Clin Exp Res 2005; 29: 1427-31.

4. Rohsenow DJ, Monti PM. Does urge to drink predict relapse after treatment?. Alcohol Res Health 1999; 23: 225-32.

5. Vocci FJ, Acri J, Elkashef A. Medication development for addictive disorders: the state of the science. Am J Psychiatry 2005;162: 1432-40.

6. O'Brien CP. Anticraving medications for relapse prevention: a possible new class of psychoactive medications. Am J Psychiatry 2005; 162: 1423-31.

7. Roberts AJ, Koob GF. The neurobiology of addiction: an overview (Abstract). Alcohol Health Res Word 1997; 21 : 101-6.

8. Tiffany ST. Cognitive concepts of craving. Alcohol Res Health 1999. 23: 215-24.

9. Tiffany ST, Conklin CA. A cognitive processing model of alcohol craving and compulsive alcohol use. Addiction 2000; 95 supl 2: S145-53.

10. Guardia J, Segura L, Gonzalvo B, Trujols J, Tejero A, Suárez A, Martí A. Validation study of the Multidimensional Alcohol Craving scale (MACS). Med Clin (Barc) 2004; 123: 211-16.
11. Morgan TJ, Morgenstern J, Blanchard KA, Labouvie E, Bux DA. Development of the OCDS--revised: a measure of alcohol and drug urges with outpatient substance abuse clients (Abstract). Psychol Addict Behav 2004; 18: 316-21.

12. Anton RF. What is craving? Models and implications for treatment. Alcohol Res Health 1999; 23: 165-73.

13. Carol G, Smelson DA, Losonczy MF, Ziedonis D. A preliminary investigation of cocaine craving among persons with and without schizophrenia. Psychiatr Serv 2001; 52: 1029-31.

14. Coffey SF, Saladin ME, Drobes DJ, Brady KT, Dansky BS, Kilpatrick DG. Trauma and substance cue reactivity in individuals with comorbid posttraumatic stress disorder and cocaine or alcohol dependence (Abstract). Drug Alcohol Depend 2002; 65: 115-27.

15. McCusker CG, Brown K. The cue-responsivity phenomenon in dependent drinkers: 'personality' vulnerability and anxiety as intervening variables (Abstract). Br J Addict 1991; 86: 905-12.

16. Salloum IM, Cornelius JR, Thase ME, Daley DC, Kirisci L, Spotts C. Naltrexone utilityin depressed alcoholics. Psychopharmacol Bull 1998; 34: 111-5.

17. Glautier S, Drummond DC. Alcohol dependence and cue reactivity (Abstract). J Stud Alcohol 1994; 55: 224-9.

18. Westerberg VS. Constituents of craving in a clinical alcohol sample (Abstract). J Subst Abuse 2000; 12 (4): 415-23.

19. Rubio Valladolid G, Urosa B, Santo-Domingo J. Validación de la escala de la intensidad de la dependencia al alcohol (EIDA). Psiquiatr Biol 1998; 5 Supl 1: 44-7.

20. Rubio G, López M. Validación del cuestionario sobre los componentes obsesivo-compulsivo de bebida en alcohólicos españoles. Adicciones 1999; 11: 7-15.

21. Drobes DJ, Thomas SE. Assessing craving for alcohol. Alcohol Res Health 1999; 23: 179-86.

22. Guardia J, Trujols J, Burguete T, Luquero E, Cardús M. The Impaired Response Inhibition Scale for Alcoholism (IRISA). (Sometido a examen en la revista Alcohol Clin Exp Res).

23. Lazcano-Ponce E, Fernández E, Salazar-Martínez E, Hernández-Ávila M. Estudios de cohorte. Metodología, sesgos y aplicación. Salud Publica Mex 2000; 42: 230-41.

24. Delgado M, Llorca J. Estudios longitudinales: concepto y particularidades. Rev Esp Salud Pública 2004; 78: 141-48. 
